\title{
Validation of the performance of a new
}

\section{bidirectional video-goniophotometer}

\author{
Marilyne Andersen ${ }^{1}, \mathrm{MScPhys}, \mathrm{PhD}$
}

Solar Energy and Building Physics Laboratory (LESO-PB)

Swiss Federal Institute of Technology (EPFL), Lausanne, Switzerland

\footnotetext{
${ }^{1}$ Address for correspondence: Prof. Marilyne Andersen, Building Technology Program, Department of Architecture, Massachusetts Institute of Technology, Room 5-418, 77 Massachusetts Avenue, Cambridge MA 02139-4307, USA. Phone: (617) 253-7714. Fax: (617) 253-6152. E-mail: mand@mit.edu
} 


\begin{abstract}
An accurate knowledge of the directional optical properties of advanced fenestration materials is necessary for them to be adequately integrated in buildings. These properties are expressed by the Bidirectional Transmission (or Reflection) Distribution Functions (BTDF, BRDF) of such elements, which are measured by specifically designed measuring equipment: an innovative, time-efficient bidirectional goniophotometer, based on digital imaging techniques, was designed and set up for that purpose.

In this paper, the in-depth validation used for the bidirectional measurements performed with this apparatus is presented. It is based on different approaches including experimental error estimation, comparisons to analytical or ray tracing based models and to other measured data, and calculation of the directionalhemispherical transmittance (reflectance) gauged against measurements of the same systems with Ulbricht (integrating) spheres. The high accuracy and reliability of this novel device were confirmed by this detailed investigation, and led to a maximum error for BT(R)DF data of only $10 \%$.
\end{abstract}




\section{List of Symbols}

$A$ sample illuminated area $\left(\mathrm{mm}^{2}\right)$

BTDF Bidirectional Transmission Distribution Function $\left(\mathrm{cd} \cdot \mathrm{m}^{-2} \cdot \mathrm{lx}^{-1}\right)$ or $\left(\mathrm{sr}^{-1}\right)$

$B R D F$ Bidirectional Reflection Distribution Function $\left(\mathrm{cd} \cdot \mathrm{m}^{-2} \cdot 1 \mathrm{x}^{-1}\right)$ or $\left(\mathrm{sr}^{-1}\right)$

$d$ distance between screen and sample centre (on emerging interface) along direction $\left(\theta_{2}, \psi_{2}\right)(\mathrm{mm})$

$D$ illuminated sample diameter (on incident interface) $(\mathrm{mm})$

$E_{1}$ illuminance on the sample plane due to the incident light flux (lx)

$e_{s}$ sample thickness (mm)

$E_{\text {screen }}$ illuminance on the screen plane (lx)

$f_{1}^{\prime}$ quantity expressing the match of $S(\lambda)$ with $V(\lambda)$

$f_{i}$ arbitrary variable referring to one of the parameters affecting the accuracy of a ray-tracing simulation

$\epsilon$ photometric error for assessing the impact of the spectral calibration

$\epsilon_{\text {elim }}$ relative error due to the elimination of pixels from a BT(R)DF measurement

$g$ gap between detection area base and external sample interface, due to mechanical constraints $(\mathrm{mm})$

$h$ distance from equivalent point source to sample (mm)

$H$ difference in level between screen detection area base and apex $(\mathrm{mm}): H=1150 \mathrm{~mm}$ 
$L_{1}$ luminance of an element of incident light flux $\left(\mathrm{cd} \cdot \mathrm{m}^{-2}\right)$

$L_{2}$ luminance of an element of emerging light flux $\left(\mathrm{cd} \cdot \mathrm{m}^{-2}\right)$

$L_{\text {screen }}$ screen luminance $\left(\mathrm{cd} \cdot \mathrm{m}^{-2}\right)$

$N_{f}$ number of individual parameters affecting the accuracy of a ray-tracing simulation

$N_{\lambda}$ number of wavelengths considered for spectral calibration

$S(\lambda)$ (corrected) spectral sensitivity of the CCD camera

$V(\lambda)$ normalized human eye's spectral sensitivity

$X, Y$ pixel co-ordinates on digital image

$\alpha$ angle between direction $\left(\theta_{2}, \psi_{2}\right)$ and normal to the screen plane (or more generally impinging angle on screen surface) $\left(^{\circ}\right)$

$\Gamma_{\text {meas }}(\lambda), \Gamma_{\text {calib }}(\lambda)$ radiometric spectra of the light source used respectively for $\mathrm{BT}(\mathrm{R}) \mathrm{DF}$ measurements and photometric calibration $\left(\mu \mathrm{W} \cdot \mathrm{mm}^{-2} \cdot \mathrm{sr}^{-1}\right)$

$\Delta \theta_{2}, \Delta \phi_{2}$ angular intervals determining the $\mathrm{BT}(\mathrm{R}) \mathrm{DF}$ averaging grid $\left(^{\circ}\right)$

$\Delta \theta_{2}^{\mathrm{rad}}, \Delta \phi_{2}^{\mathrm{rad}}$ angular intervals determining the BT(R)DF averaging grid (rad)

$\Delta \lambda_{n}$ wavelength interval between measured data $(n-1)$ and $n(\mathrm{~nm})$

$\varepsilon_{\text {elim }}$ relative error due to eliminated pixels along cover edges

$\varepsilon_{\mathrm{BT}(\mathrm{R}) \mathrm{DF}}$ relative error on final $\mathrm{BT}(\mathrm{R}) \mathrm{DF}$ values

$\varepsilon_{\tau_{\mathrm{dh}} \mid \rho_{\mathrm{dh}}}$ relative error on $\tau_{\mathrm{dh}}$ or $\rho_{\mathrm{dh}}$ values 
$\Theta_{0}$ projection screen inclination angle regarding the sample plane $\left(^{\circ}\right): \Theta_{0}=\arctan \frac{2}{\sqrt{3}} \cong$ $49.1^{\circ}$

$\theta_{1}, \phi_{1}$ polar co-ordinates of the incident light flux $\left(^{\circ}\right)$

$\theta_{2}, \phi_{2}$ polar co-ordinates of the emerging (either transmitted or reflected) light flux $\left({ }^{\circ}\right)$

$\lambda$ wavelength (nm)

$\rho_{\mathrm{dh}}$ directional-hemispherical light reflectance, for a given incident direction

$\rho_{\text {dir }}$ regular (directional-directional) light reflectance, for a given incident direction

$\rho_{\text {screen }}$ screen reflection coefficient

$\tau_{\mathrm{dh}}$ directional-hemispherical light transmittance of sample, for a given incident direction

$\tau_{\mathrm{dh}} \mid \rho_{\mathrm{dh}}$ combined symbol to express either $\tau_{\mathrm{dh}}$ or $\rho_{\mathrm{dh}}$

$\Upsilon_{\theta_{2}}, \Upsilon_{\psi_{2}}$ maximum divergence of emerging rays reaching a given point on the projection screen $\left(^{\circ}\right)$

$\psi_{2}$ azimuth angle projected on the sample plane and lying between $-30^{\circ}$ and $+30^{\circ}$ (zero along the central axis of the screen), for an arbitrary screen position $\left({ }^{\circ}\right)$

$d \omega_{1}, d \omega_{2}$ solid angle subtended by the incident, emerging light flux (sr) 


\section{Introduction}

In the present context of increasing needs for sustainability and energy savings, reducing the environmental impact of buildings positions itself as a priority. As a consequence, the control of daylight and solar radiation through fenestration systems has received a growing attention both in research and practice ${ }^{1,2}$. Advanced fenestration systems, which include solar blinds, new glazing materials and daylight redirecting devices, can contribute to reducing significantly the energy consumption of buildings, while simultaneously improving visual comfort conditions for users ${ }^{3}$. Full knowledge of the light distribution characteristics of such systems is, however, required to control the propagation of daylight deeper in rooms and to allow larger solar gains in winter and lower solar loads in summer ${ }^{4}$.

Such characteristics are expressed in terms of Bidirectional Transmission (or Reflection) Distribution Functions, abbreviated BT(R)DFs, defined by the Commission Internationale de l'Eclairage ${ }^{5}$ as the quotient of the luminance of a surface element in a given direction by the illuminance incident on the material. These values depend on both the angles of incidence and of emergence.

To assess these functions for complex fenestration systems, several bidirectional goniophotometers have been developed $\mathrm{d}^{6,7,8,9,10}$. Most are based on a conventional design using a movable photo-sensor to track and measure in all possible directions the luminance of the sample, after either transmission or reflection. Their performance is accurate and reliable, but they show two significant drawbacks: on one hand they are time consuming because numerous movements of the mobile photo-sensor are required to achieve an appropriate angular resolution, the number of these movements increasing tremendously with a finer resolution; 
on the other hand, the risk of missing a narrow light peak with a large gradient in the space left between two measurement points can never be avoided. Materials with a high dynamical luminance range can also cause serious technical difficulties, and require local refinements of the angular resolution.

To overcome these problems, an innovative type of bidirectional goniophotometer was developed ${ }^{11,12}$, based on the projection of emerging light on a diffusing screen before being detected by a calibrated CCD camera, used as a multiplepoint luminance meter. This detection principle allows the time needed to monitor BT(R)DF data to be reduced to a few minutes per incident direction instead of the several hours required for conventional methods based on a scanning process.

As for any new measurement device, validation of the results it produces is a crucial issue. As there are no standard procedures for validating goniophotometric data of complex fenestration materials, different approaches were chosen:

- assessment of the experimental uncertainty at each intermediate stage of calibration and processing, leading to a global maximum error for BT(R)DFs;

- bidirectional measurement of systems presenting a known symmetry, and verification against standard luminance meter data or analytical calculations;

- empirical validation based on bidirectional measurement comparisons between different devices; when there is disagreement, however, no conclusion can be established;

- assessment of hemispherical optical properties by integrating BT(R)DF 
data over the whole hemisphere and comparison to integrating sphere measurements;

- comparison of monitored data with ray tracing simulations to achieve a higher level of detail in the assessment of BT(R)DF behaviour.

At least one of the first four methods has been applied to other existing goniophotometers $13,14,10,6$. In this paper, the validation results obtained with all five approaches are presented.

\section{BT(R)DF assessment method}

The novel bidirectional video-goniophotometer considered in this paper is extensively described in two papers by Andersen et al. ${ }^{11,12}$. Its functioning principle is briefly summarized in this section.

Unlike other goniophotometers for measuring BT(R)DF's, this instrument has a flat, triangular diffusing screen which collects the light from the sample and at which a calibrated Charge-Coupled Device (CCD) camera is aimed. To cover all possible directions of the emerging light ( $2 \pi$ steradian), the camera and the screen perform six rotations of a $60^{\circ}$ angle magnitude, leading to the investigation of the full light flux transmission or reflection figure (Figure 1).

For BRDF measurements, there is one position (all six for normal incidence) where the screen obstructs the incoming light flux. In this case, a special opening in the latter is required to let the beam reach the sample, producing a blind spot at that specific screen position. As explained in the paper by Andersen et al. ${ }^{12}$, nine elliptic holes were therefore cut out of the screen to let the beam pass through every $10^{\circ}$ in altitude (the removal and repositioning of the covers for these holes 
is taken care of by a robot). Darker pixels were observed in the images along the edges of these covers, which were eliminated from the measurements as discussed in Section 3.2.

This original assessment method therefore avoids the need for point-by-point scanning of the emerging light by a moving sensor, which reduces the measurement time by a factor of about a hundred or more, depending on the resolution chosen for the scanning. In addition, it overcomes the risk of missing a discontinuity in the emerging luminance distribution. Instead, it splits the collecting hemisphere into a regular grid of averaging sectors, illustrated in Figure 1, allowing the production of a finite set of BT(R)DF data. Each BT(R)DF value is associated with four angles $\left(\theta_{1}, \phi_{1}, \theta_{2}, \phi_{2}\right)$ where $\theta_{1}, \phi_{1}$ and $\theta_{2}, \phi_{2}$ are the polar co-ordinates of the incident and emerging (either transmitted or reflected) light flux respectively, all expressed in $\left(^{\circ}\right)$ and shown in Figure 2 with the luminances $L_{1}$ and $L_{2}$ and the solid angle elements $d \omega_{1}$ and $d \omega_{2}$ they are associated with. Hence, there are no gaps $\Delta \theta_{2}$ and $\Delta \phi_{2}$ between two data points, as would be the case in a conventional scanning process, where interpolation is implicit. Here, $\Delta \theta_{2}$ and $\Delta \phi_{2}$ give the angular distance between two averaging sectors centres $\left(\theta_{2}, \phi_{2}\right)$ and $\left(\theta_{2}, \phi_{2} \pm \Delta \phi_{2}\right)$ or $\left(\theta_{2} \pm \Delta \theta_{2}, \phi_{2}\right)$, which all truly represent adjacent hemisphere portions. The BT(R)DF investigation is therefore continuous, the only limit in resolution being determined by the pixellisation of the digital images.

A continuous investigation is critical especially for materials presenting a highly dynamic luminance range. These require local refinements of the angular resolution as well as a preliminary investigation for scanning-based characterization methods. 
Figure 1: Light detection principle in transmission (BTDF) and reflection (BRDF) modes for the LESO-PB / EPFL video-goniophotometer.

Figure 2: Photometric and geometric quantities for BT(R)DF definition.

To be used as a multiple-point luminance meter, the overall video digital system must follow detailed calibration and correction procedures. These probably constitute the main difficulty of this approach compared to the conventional moving sensor approach, which provides the desired output almost directly. The reliability of the data assessment and its related accuracy depend on the careful execution of these procedures ${ }^{11,15}$. They include: the spectral calibration of the CCD video camera, so that its response matches that of the human eye's sensitivity $V(\lambda)^{16}$ as closely as possible; the conversion of greyscale levels (or more generally speaking the pixel values) into luminance values, for each integration interval; and the determination of the relation between the pixel co-ordinates of the image and the corresponding light ray direction ${ }^{17}$.

Additionally, to avoid losses of accuracy in the BT(R)DF assessment, other possible sources of experimental error were checked and, whenever possible, compensated for or corrected analytically ${ }^{15}$ : the incident beam collimation, the uniformity of the pixels' response and stray light, mainly due to the incident beam penetration in BRDF mode.

Once fully calibrated, the camera can be used to carry out luminance mapping of the projection screen by capturing images of it at different integration intervals, thus avoiding over and under-exposure effects. The latter are then treated 
appropriately to extract $\mathrm{BT}(\mathrm{R}) \mathrm{DF}$ data: the direction of emergence $\left(\theta_{2}, \phi_{2}\right)$ and luminance $L_{\text {screen }}$, with which each pixel is associated, are determined. Based on these parameters and using the simultaneously measured sample illuminance $E_{1}\left(\theta_{1}\right)$, the corresponding BT(R)DF values are calculated from Equation $(1)^{11}$ at the pixel level (cf. list of symbols for quantities definition) and gathered according to a suitable averaging grid $^{17,18}$ (Figure 3).

$$
B T(R) D F\left(\theta_{1}, \phi_{1}, \theta_{2}, \phi_{2}\right)=\frac{\pi}{\rho_{\text {screen }}} \cdot \frac{d^{2}\left(\theta_{2}, \psi_{2}\right)}{A \cdot \cos \alpha \cdot \cos \theta_{2}} \cdot \frac{L_{\text {screen }}\left(\theta_{1}, \phi_{1}, \theta_{2}, \phi_{2}\right)}{E_{1}\left(\theta_{1}\right)}
$$

Figure 3: Conversion from $L_{\text {screen }}$ mappings into BT(R)DF data.

\section{Experimental error}

In this section, the sources of error caused by the various calibration and correction procedures, as well as the processing or mechanical constraints, are analyzed. In the following sections, different approaches for validation are presented, based on analytical or simulation models or empirical comparisons.

\subsection{Calibration and correction error sources}

The spectral response of the CCD camera was first determined experimentally, then corrected by a combination of optical filters ${ }^{11}$. The relative error $f_{1}^{\prime}$ defined by the Commission Internationale de l'Eclairage ${ }^{19}$ determines how well the relative spectral sensitivity $S(\lambda)$ of the CCD camera matches the photopic function $V(\lambda)$. Its expression and applicable approximation are given by Equation (2):

$$
f_{1}^{\prime}(-)=\frac{\int_{0}^{\infty}|S(\lambda)-V(\lambda)| d \lambda}{\int_{0}^{\infty} V(\lambda) d \lambda} \simeq 0.0093584 \cdot \sum_{n=1}^{N_{\lambda}}\left|S\left(\lambda_{n}\right)-V\left(\lambda_{n}\right)\right| \cdot \Delta \lambda_{n}
$$


$f_{1}^{\prime}$ was found to be equal to $10.2 \%$. Its actual effect on the BT(R)DF measurements is, however, by far lower than $10 \%$, as it only applies as a secondary error source. Indeed, as the photometric calibration determines the relation between pixel greyscale value and luminance taking the camera's sensitivity $S(\lambda)$ into account, results will be affected only if the sources used for calibration and measurement present different spectra: the closer $S(\lambda)$ is to $V(\lambda)$, the less these spectrum differences will matter.

To quantify the extent to which these differences will influence the results, the photometric error $\epsilon$ is used ${ }^{19}$, given by Equation (3):

$$
\epsilon(\%)=\frac{\left(\sum_{n=1}^{N_{\lambda}} S\left(\lambda_{n}\right) \cdot \Gamma_{\text {meas }}\left(\lambda_{n}\right) \cdot \Delta \lambda_{n}\right) \cdot\left(\sum_{n=1}^{N_{\lambda}} V\left(\lambda_{n}\right) \cdot \Gamma_{\text {calib }}\left(\lambda_{n}\right) \cdot \Delta \lambda_{n}\right)}{\left(\sum_{n=1}^{N_{\lambda}} V\left(\lambda_{n}\right) \cdot \Gamma_{\text {meas }}\left(\lambda_{n}\right) \cdot \Delta \lambda_{n}\right) \cdot\left(\sum_{n=1}^{N_{\lambda}} S\left(\lambda_{n}\right) \cdot \Gamma_{\text {calib }}\left(\lambda_{n}\right) \cdot \Delta \lambda_{n}\right)}
$$

As the measurement source itself was used for calibration, $\epsilon$ can be neglected, as it only depends on the source's own fluctuations in spectrum of about $0.6 \%$; when assessed against the solar spectrum, the photometric error does not go beyond $0.6 \%$.

One important restriction of the BT(R)DF data's appositeness should be outlined. Owing to its photometric nature, a spectrally selective sample (coloured or coated glass, film, screen) will not be differentiable from a neutral but less transmissive (reflective) one if the total loss in emerging luminance is the same. This implies that no conclusion can be made on the BT(R)DF resulting from a superposition of possibly selective layers if these were only assessed individually (thus requiring them to be measured again in their superposed configuration).

The photometric calibration consisted of capturing images of a diffusing surface 
at all possible integration intervals used for sample measurements for a varying illumination situation and relating the obtained greyscale values to the simultaneously measured luminances assessed with a validated luminance meter. The latter showed variations, which were very low: for usual luminances, an average relative fluctuation of $0.3 \%$ was observed, which remained below $2 \%$ for the lowest measured values. As far as the analyzed area on the diffusing surface is concerned, the standard deviation in greyscale level of the pixels was less than $1 \%$ in relative terms, which implies they differed on average by less than 1 absolute level.

Spatial calibration (which defines the relation between pixel coordinates $(X, Y)$ and emerging directions $\left.\left(\theta_{2}, \phi_{2}\right)\right)$ was shown to have no significant effect on BT(R)DF accuracy.

The sample illuminance was measured simultaneously with each image capture by an external luxmeter, over a period of time corresponding to the image's integration interval. The measured illuminance values were found to have a relative inaccuracy of less than $1 \%$ while the illuminance uniformity over the sample area showed $1.4 \%$ relative variation $\left(2 \%\right.$ for grazing angles $\left.\theta_{1}>70^{\circ}\right)$.

To compensate for the spread of the incident beam reaching the sample area $A$, correction factors were estimated and applied to the sample diameter $D^{15}$. These factors showed a relative error of $1.4 \%$, which therefore gives a $2 \%$ relative error $\left(\frac{\Delta A}{A}=\sqrt{2} \frac{\Delta D}{D}\right)$ for the area.

As a darkening of pixels was observed towards the edges of the images, a cor- 
rection was applied by estimating the factor to be applied to each pixel ${ }^{15}$; these factors were determined with a $0.6 \%$ relative error.

Stray light was made negligible by choosing highly absorbing materials for the goniophotometer's inner surfaces, which were therefore covered with "velvetine" (highly absorbing, black velvet-like material), and by performing the measurements in a dark chamber. No influence of internal reflections could be detected, whereas some detectable stray light was caused by the light rays passing through the obstructing screen position in reflection mode. This excess light flux was however compensated for by subtraction of an adequately calibrated background image, leading to a negligible error due to stray light.

\subsection{Inaccuracies due to equipment constraints}

The captured digital images were treated as maps of luminances to be converted into BT(R)DF data. Several parameters influence the quality of the achieved maps.

Pixel resolution was shown to affect the data by only $0.5 \%$. This value was assessed by using ray tracing calculations: BT(R)DF data based on a faithful model of the averaging grid were compared to values obtained with a shifted one (by the equivalent in degrees of half a pixel in altitude and azimuth i.e. the average extent of a pixel in both directions ${ }^{15}$ ).

The blind zone induced in reflection mode by the hole made for the passage of the incident beam for one of the screen positions must not be considered as an error source in itself but as missing information, which does not influence the quality 
of the monitored BT(R)DF data.

Eliminating the pixels corresponding to the edges of the screen covers, however (see Section 2), does affect BT(R)DFs as it requires an implicit interpolation of neighbouring pixels. To estimate the degree to which this retrieval influences the final data, bidirectional transmission measurements made with a full projection screen (presenting no cut-out ellipses) were compared with those obtained for the same samples using the screen shown in Figure 4. Overall, a standard deviation of $5 \%$ due to the effect of eliminated pixels was found.

Figure 4: Screen image: the edges of the elliptic covers generate a slight darkening of the pixels, which are therefore eliminated from the BT(R)DF assessment.

Each surface element on the detection screen (i.e. each image pixel), although associated with a single (average) emerging direction $\left(\theta_{2}, \psi_{2}\right)$, can be reached by rays corresponding to a range of directions, the extent of which is proportional to the sample illuminated area.

As BT(R)DFs are averaged within sectors the angular extent of which is proportional to the sample size, this possible divergence is compensated for as long as angular intervals $\Delta \theta_{2}$ and $\Delta \phi_{2}$ are greater or equal to angular ranges $\Upsilon_{\theta_{2}}$ and $\Upsilon_{\psi_{2}}$ for altitude and azimuth respectively. Since both $\Upsilon_{\theta_{2}}$ and $\Upsilon_{\psi_{2}}$ are functions of $\theta_{2}$ and $\phi_{2}$ and thus vary over the screen area, this compensation needs to be checked for every combination of direction $\left(\theta_{2}, \phi_{2}\right)$, thickness $e_{s}$ (affecting $d$ ) and sample diameter $D$, as $D$ determines the optimum intervals $\Delta \theta_{2}$ and $\Delta \phi_{2}$ (typically equal to $\left(5^{\circ}, 5^{\circ}\right),\left(10^{\circ}, 15^{\circ}\right)$ and $\left(15^{\circ}, 20^{\circ}\right)$ for $D=100 \mathrm{~mm}, 200 \mathrm{~mm}$ and $300 \mathrm{~mm}$ respectively). Fortunately, the study revealed that such values remained greater than the corresponding $\Upsilon_{\theta_{2}}$ and $\Upsilon_{\psi_{2}}$ values for almost all of these 
combinations.

The screen diffusion variations were assessed experimentally for different impinging and emerging directions and found to be equal to $2.6 \%$. Accounting also for the slight spectral dependence of the reflection coefficient $\rho_{\text {screen }}$ over the visible range (1.5\%) and for its $0.2 \%$ variation over the surface of the screen, a relative uncertainty of $3 \%\left(\sqrt{0.026^{2}+0.015^{2}+0.002^{2}}=0.0301\right)$ was found.

The light source remaining fixed, the incident direction is determined by tilting and rotating the sample itself. The uncertainty on the tilt angle was observed to lie between $0.1^{\circ}$ and $0.2^{\circ}$; the sample holder's positioning accuracy was checked to be of $\pm 0.2^{\circ}$.

Ray tracing simulations were then used to estimate the influence on BT(R)DF data of variations in incident altitude and azimuth of $\pm 0.15^{\circ}$ and $\pm 0.2^{\circ}$ respectively. The resulting mean relative BTDF fluctuations were of $1.3 \%$ and $0.3 \%$, leading to a standard deviation of about $1.3 \%\left(\sqrt{0.013^{2}+0.003^{2}}=0.0133\right)$ due to incident direction inaccuracy.

The projection screen's positioning uncertainty with respect to the sample plane were measured with a micrometric protractor, a spirit level and source beam alignment. They were found to be equal to about $\pm 0.1^{\circ}$. Using again ray-tracing simulations and comparing slightly shifted screen situations, a standard deviation of $0.8 \%$ was determined on BTDFs. As this error is associated with the distance $d$ from sample to screen, its actual effect on BT(R)DFs is doubled, $d$ being squared in Equation (1). 


\subsection{Global relative error}

Table 1 gathers all the significant error sources from the above analysis and associates each of them to the affected parameter in Equation (1).

Table 1: Relative errors incurred at the different calibration stages and list of corresponding affected parameters in Equation (1).

The global maximum error $\varepsilon_{\mathrm{BT}(\mathrm{R}) \mathrm{DF}}$ in the monitored BT(R)DF data was calculated from Equation (4), $\varepsilon_{\text {elim }}$ being added separately because it affects $B T(R) D F$ values directly:

$$
\varepsilon_{\mathrm{BT}(\mathrm{R}) \mathrm{DF}}=\frac{\Delta B T(R) D F}{B T(R) D F}=\sqrt{\sum_{i=1}^{N_{f}}\left(\frac{\Delta f_{i}}{f_{i}}\right)^{2}}+\varepsilon_{\mathrm{elim}}
$$

By including the different specific terms of Table 1 in Equation (4), a global maximum error for BT(R)DF data was determined:

$$
\varepsilon_{\mathrm{BT}(\mathrm{R}) \mathrm{DF}}=4.5 \%+5.0 \% \simeq 10 \%
$$

\section{Analytical verification}

Known symmetries were checked for the corresponding BT(R)DF data sets for various materials, amongst which a white opalescent acrylic panel and the highly diffusing coating of the projection screen $(2.6 \%$ relative variations from the lambertian model, see Section 3.2), manufactured by LMT $^{\circledR}($ LMT Lichtmesstechnik GmbH Berlin).

For the first diffuser, rotational symmetry was checked in transmission at the pixel 
level and found to present $1.2 \%$ relative fluctuations, the deviation from a lambertian distribution being $3.1 \%$ for all non-grazing directions. In reflection, a strong specular component of predictable direction $\left(\theta_{2}, \phi_{2}\right)=\left(\theta_{1}, \phi_{1}+180^{\circ}\right)$ combined with a diffuse behaviour in all other directions was expected and observed, the isotropic diffuse distribution being verified with a $1.5 \%$ relative fluctuation.

For the second, the observed fluctuations for all $\theta_{2}$ and $\phi_{2}$ angles remained close to $5.4 \%$ and the total reflectance $\rho_{\mathrm{dh}}$ deduced from measured BRDF data (see Equation (7)) was checked against the $\rho_{\text {screen }}=0.75$ value assessed with a Minolta CR-200b surface chromameter. $\rho_{\mathrm{dh}}$ was found equal to 0.81 , leading to a $8 \%$ relative discrepancy.

With a simple aperture $\left(\tau_{\mathrm{dh}}=1\right)$, an expression for $\left(\frac{L_{\text {screen }}}{E_{1}}\right)$ within the directly illuminated screen area (unique bright spot) can easily be deduced; it is given by Equation (6), where $h$ is the distance from the sample to a point source that would provide an equivalent beam spread:

$$
\frac{L_{\text {screen }_{\text {spot }}}}{E_{1}\left(\theta_{1}\right)}=\frac{\rho_{\text {screen }}}{\pi} \frac{h^{2} \cdot \cos \alpha}{(h+d)^{2}}
$$

A set of theoretical values were thus established for different $\theta_{1}$ and $D$ configurations, as well as for intermediate screen positions (to vary $d$ and $\alpha$ ). They were compared to the ones obtained for the calibrated pixels within the corresponding bright spots.

The comparison showed, again, a very good agreement between theory and measurement: the relative differences were lower than $8.7 \%$ with an average of only $2.9 \%$ for the configurations considered. 


\section{Empirical validation}

Some early stage comparisons of BTDF data monitored with several existing goniophotometers were carried out within the framework of International Energy Agency Task 21 "Daylighting in Buildings"6; unfortunately, only a few bidirectional datasets were made available, mainly from Berlin University of Technology (TUB), in addition to the extensive set of EPFL data ${ }^{15}$.

Based on these other goniophotometric data, BT(R)DF comparisons were made for three types of glazing. Because of the important differences in the assessment methods (mean BT(R)DFs inside adjacent hemisphere parts versus scanning process), such comparisons would not be valid for steep luminance gradients; systems presenting diffuse components in their emerging light distribution were thus selected.

Opalescent glazing had been measured within the REVIS project conducted by the TNO Building and Construction Research in The Netherlands ${ }^{20}$ and a good general agreement between the two datasets was found, with relative differences of only $5 \%$.

To conduct comparisons for another opalescent acrylic panel manufactured by Roehm, TUB's datasets were first averaged inside angular sectors corresponding to the EPFL measurements, and results compared for all incident directions individually (see Table 2).

Both datasets fit each other within a very reasonable range: for all $\left(\theta_{1}, \theta_{2}, \phi_{2}\right)$ configurations, a mean relative difference of $8.1 \%$ was found, reducing to $6.2 \%$ if only non-grazing $\theta_{2}$ angles are considered $\left(\theta_{2}<70^{\circ}\right)$. This demonstrates on the 
one hand the reliability of both devices and on the other hand that the averaging method applied for the EPFL goniophotometer does not alter the quality of the measurements.

Table 2: Mean relative differences between TUB and EPFL measurements for the BTDF characterization of the opalescent acrylic for different incident altitudes $\theta_{1}$.

Finally, the sun-directing glass "Lumitop ${ }^{\mathrm{TM}}$ " manufactured by Vegla was considered for a BTDF comparison.

From the available set of 145 incident directions, incidence $\left(\theta_{1}, \phi_{1}\right)=\left(60^{\circ}, 285^{\circ}\right)$ was selected for the significant scattering features presented by its transmission figure; the latter were located on the hemispherical projection shown on Figure 5(a).

The monitored BTDFs are shown on Figures 5(b) and 5(c) for the two laboratories. The discrete TUB data along directions $\left(\theta_{2}, \phi_{2}\right)$ were averaged inside angular sectors of intervals $\left(\Delta \theta_{2}, \Delta \phi_{2}\right)=\left(5^{\circ}, 5^{\circ}\right)$, in accordance with the EPFL investigation method.

For the selected diffuse space portions, the data were then compared: although the transmission features were this time not as close to the lambertian model as for the opalescent acrylic, only a 3.5\% mean relative difference was found between the two datasets (with a maximum divergence of $8.5 \%$ ), which strongly reinforces the previous conclusions for diffusing materials.

Figure 5: BTDF data $\left(\mathrm{cd} \cdot \mathrm{m}^{-2} \cdot \mathrm{lx}^{-1}\right)$ monitored by TUB and EPFL for the sun directing glass, incidence $\left(\theta_{1}, \phi_{1}\right)=\left(60^{\circ}, 285^{\circ}\right)$; (a) Projection of BTDF data on virtual hemisphere: 
quasi-diffuse transmission sectors located in circles, (b) TUB photometric solid, (c) EPFL photometric solid.

\section{Directional-hemispherical transmittance (reflectance) comparisons}

Numerically integrating bidirectional data over the whole emerging hemisphere to compare directional-hemispherical transmittance or reflectance values is one of the most commonly used methods to validate BT(R)DFs measurements $21,8,10,22,20$.

The mathematical development leading from BT(R)DF data to the directionalhemispherical visible transmittance $\tau_{\mathrm{dh}}\left(\theta_{1}, \phi_{1}\right)$ or reflectance $\rho_{\mathrm{dh}}\left(\theta_{1}, \phi_{1}\right)$, expressed by the combined symbol $\tau_{\mathrm{dh}} \mid \rho_{\mathrm{dh}}$ for simplification, can be found in ${ }^{15}$; the relation obtained is given by Equation (7), which agrees with the relation provided for $\tau_{\mathrm{dh}}$ in the source book of the International Energy Agency, Task $21^{6}$ :

$\tau_{\mathrm{dh}} \mid \rho_{\mathrm{dh}}\left(\theta_{1}, \phi_{1}\right)=\frac{1}{2} \cdot \Delta \theta_{2}^{\mathrm{rad}} \cdot \Delta \phi_{2}^{\mathrm{rad}} \cdot \sum_{\theta_{2}=0}^{\theta_{2_{\max }}} \sum_{\phi_{2}=0}^{360^{\circ}-\Delta \phi_{2}} B T(R) D F\left(\theta_{1}, \phi_{1}, \theta_{2}, \phi_{2}\right) \cdot \sin \left(2 \theta_{2}\right)$

To estimate the inaccuracy of $\tau_{\mathrm{dh}}$ or $\rho_{\mathrm{dh}}$ values deduced from BT(R)DFs, the calculation of the square root of the summed squared individual uncertainties for BT(R)DFs is a far too pessimistic method, which leads to increasing errors when the averaging grid intervals $\Delta \theta_{2}$ and $\Delta \phi_{2}$ decrease: this disagrees with the fact that the sum (7) will actually become a better approximation of the integral from which it is deduced. In consequence, a different approach was adopted to eval- 
uate these errors, based on comparisons with either calculated or experimentally assessed $\tau_{\mathrm{dh}}$ or $\rho_{\mathrm{dh}}$ values.

For simple apertures of various diameters $D(100 \mathrm{~mm}, 150 \mathrm{~mm}$ and $200 \mathrm{~mm})$, transmittances $\tau_{\mathrm{dh}}$ determined through Equation (7) were compared to their expected value of 1 for incident altitudes $\theta_{1}$ between $0^{\circ}$ and $75^{\circ}$.

About twenty $D$ and $\theta_{1}$ configurations were considered, showing discrepancies of $5 \%$ on average and $15 \%$ at most, all but two being less than $10 \%$. No correlation with either the diameter $D$ or the altitude $\theta_{1}$ was found, confirming the accuracy of the $\tau_{\mathrm{dh}}\left(\right.$ or $\left.\rho_{\mathrm{dh}}\right)$ calculation method.

Based on these results, a range of $10 \%$ to $15 \%$ was determined as representative of the error on directional-hemispherical transmittance or reflectance.

To refine this statement and to validate the BT(R)DFs themselves, $\tau_{\mathrm{dh}}$ and $\rho_{\mathrm{dh}}$ values based on goniophotometric data were compared to Ulbricht (integrating) sphere $^{23}$ measurements carried out by different European institutes ${ }^{1}$ for several optical materials. These materials included an opalescent acrylic panel, a laser cut panel, a prismatic film manufactured by $3 \mathrm{M}$, an acrylic prism manufactured by Siemens AG presenting $42^{\circ} / 5^{\circ}$ gratings (asymmetric panel), two coated fabric blinds and a white opalescent plastic panel.

The measured $\tau_{\mathrm{dh}} \mid \rho_{\mathrm{dh}}$ values were compared for these different systems with those obtained with the goniophotometer. Some of the results are shown in Figures 6 and 7.

\footnotetext{
${ }^{1}$ Berlin University of Technology (TUB), Fraunhofer Institute for Solar Energy Systems (ISE) and Siemens Lighting Division in Germany; Bartenbach Lichtlabor (BAL) in Austria; Building and Construction Research Organisation (TNO) in The Netherlands; Glaceries de Saint-Roch (GSR), Centre de développement du Bâtiment, in France; Stazione Sperimentale di Vetro (SSV), Optical Testing Laboratory and Istituto Elettrotecnico Nazionale Galileo Ferraris (IENGF) in Italy; the Solar Energy and Building Physics Laboratory (LESO-PB) at EPFL, in Switzerland.
} 
From the discrepancies observed on measured $\tau_{\mathrm{dh}} \mid \rho_{\mathrm{dh}}$ values between laboratories for a same material, standard deviations were calculated for each incident direction and were used as as estimation of their error (error bars for "Integrating Spheres" curves). The error bars for the "Goniophotometer" curves of Figures 6 and 7 correspond to the relative maximum error of $15 \%$ determined above.

Figure 6: Directional-hemispherical light transmittance comparisons for diffusing and redirecting glazings: $\tau_{\mathrm{dh}}$ values based on BTDF integration compared to Ulbricht sphere measurements for (a) the white opalescent acrylic and (b) the Laser Cut Panel.

Figure 7: Directional-hemispherical light transmittance comparisons for a 3M prismatic film on float glass: $\tau_{\mathrm{dh}}$ values based on BTDF integration compared to Ulbricht sphere measurements for the $3 \mathrm{M}$ film on the (a) incidence side and (b) emerging side.

If we consider the systems individually, we can observe that for very diffuse materials (opalescent plastics), the discrepancies remain generally lower than $6 \%$ in relative terms. For strongly specular systems such as the laser cut panel (Figure $6(b)$ ), the Siemens prisms and the grey fabric blind, the mean relative difference was of about $14 \%$, but the integrating sphere data showed variations for these materials that were actually even larger. Finally, for hybrid systems like the $3 \mathrm{M}$ prismatic film (Figure 7) and the grey and red fabric blind that both present high luminance gradients combined with a smooth light scattering for many incident directions, a mean relative difference of $13 \%$ was observed, despite, again, there being very large differences in the integrating sphere data for the $3 \mathrm{M}$ prismatic film. 
As a consequence, this analysis confirmed the accuracy of the BT(R)DF measurements and the adequacy of the $\tau_{\mathrm{dh}} \mid \rho_{\mathrm{dh}}$ calculation method; it also confirmed that the maximum errors of respectively $10 \%$ and $15 \%$ were applicable for any system type.

\section{Detailed BT(R)DF comparisons with ray tracing simulations}

In Andersen et al. ${ }^{24,25}$, the reader can find the description of a novel validation methodology for detailed bidirectional properties of a complex system with ray tracing simulations. It was applied on a substantial set of experimental BT(R)DF data for different fenestration systems and led to relative discrepancies between the two approaches far less than the assumed inaccuracies of the simulation results themselves.

\section{Conclusion}

In this paper, a detailed assessment of the experimental accuracy of an innovative goniophotometer based on digital imaging techniques was carried out using different approaches.

The many intermediate calibration stages necessary to extract BT(R)DF data from raw digital images are reviewed and the experimental error associated with each of them calculated. An overall maximum error for BT(R)DF data equal to $10 \%$ was estimated and thereafter verified with detailed comparisons using different approaches. This error corresponds to a very satisfactory accuracy for measurements as complex as BT(R)DFs, considering that even commercial illuminance meters and luminance meters (used as references for calibration) can show errors between $3 \%$ and $6 \%$ and between $4 \%$ and $7 \%$ respectively ${ }^{19}$ and that 
a BT(R)DF is equal to the ratio of a luminance and an illuminance value.

Different materials presenting predictable transmission or reflection features were characterized; expected BTDF or BRDF values were verified against measured data, with discrepancies varying from $1 \%$ to $8 \%$.

Within the framework of international projects, several systems were characterized with various goniophotometers. Whenever possible, the respectively obtained bidirectional measurements were compared for diffuse transmission with measurements taken with the present device. These comparisons showed differences lower than $8 \%$, which verified that the $\mathrm{BT}(\mathrm{R}) \mathrm{DF}$ data were reliable for the different instruments. It also showed that the averaging method chosen for this instrument did not alter the quality of the results.

The more commonly used method for validating BT(R)DF data was also applied to cross-check the reliability and accuracy of the novel goniophotometer. It consisted of integrating numerically the bidirectional data to deduce the directionalhemispherical transmittance or reflectance. This value was then compared to an Ulbricht sphere measurement made with the same sample at various European laboratories. Overall, the agreement between the two assessment methods proved excellent, the observed discrepancies remaining lower than the measurement errors.

These results, together with an analysis performed in the absence of any sample, allowed the determination of a relative maximum error of $15 \%$ on the $\tau_{\mathrm{dh}}$ and $\rho_{\mathrm{dh}}$ values deduced from BT(R)DF data.

Overall, these different validation approaches, in addition to the more detailed 
ray-tracing simulations comparison carried out by Andersen et al. ${ }^{24,25}$, led to satisfactory results for the bidirectional goniophotometer described in this paper, placing reliance on the assumptions made in the construction of the instrument and on the various calibration and correction procedures that were necessary to convert the CCD camera into a reliable multiple-points luminance meter.

The growing interest shown by practitioners and the window components industry in this kind of data confirms the adequacy of this measurement device for fulfilling their needs. It allows them to benefit from an easy, rapid - and therefore cheap - access to reliable and accurate characterization of their products and prototypes. It also provides them with objective guidelines for improving their performance, as suitable adjustments of shapes, coatings or both (reflection coefficients, texture, etc.) can be recommended, and thereafter verified for further optimization.

In the field of building simulation tools, the potentialities of daylighting performance assessment programs will be considerably increased if they can benefit from an accurate and objective database of new fenestration systems and include their bidirectional properties in the calculation routines.

\section{Acknowledgements}

This work was supported by the Swiss Federal Institute of Technology in Lausanne (EPFL) and the Commission for Technology and Innovation (CTI) of the Swiss Federal Government. The author wishes to thank Lambda Research Corporation for having provided her with a license of TracePro ${ }^{\circledR}$ to complete this research. 


\section{References}

[1] N. Baker, A. Fanchiotti, and K. Steemers. Daylighting in Architecture - A European Reference Book. James \& James, London, 1993.

[2] P.J. Greenup, J.M. Bell, and I. Moore. The importance of interior daylight distribution on overall energy performance. Renewable Energy, 22:45-52, 2001.

[3] M. Kischkoweit-Lopin. An overview of daylighting systems. Solar Energy, 73(2):7782, 2002.

[4] J. Karlsson, M. Rubin, and A. Roos. Evaluation of predictive models for the angle-dependent total solar energy transmittance of glazing materials. Solar Energy, 71(1):23-31, 2001.

[5] Commission Internationale de l'Eclairage. Radiometric and photometric characteristics of materials and their measurement. CIE, 38(TC-2.3), 1977.

[6] International Energy Agency. Daylight in Buildings - A source book on daylighting systems and components. IEA SHC Task 21 / ECBCS Annex 29, Berkeley, July 2000.

[7] K.M. Papamichael, J. Klems, and S. Selkowitz. Determination and Application of Bidirectional Solar-Optical Properties of Fenestration Materials. Technical Report LBL-25124, Lawrence Berkeley National Laboratory, Berkeley, 1988.

[8] P. Apian-Bennewitz. Designing an apparatus for measuring bidirectional reflection/transmission. In Proceedings SPIE Vol. 2255, Optical Materials 
Technology for Energy Efficiency and Solar Energy Conversion XIII, pages 697-706, 1994.

[9] L. Bakker and D. van Dijk. Measuring and processing optical transmission distribution functions of TI-materials. Private Communication, TNO Building and Construction Research, Delft, 1995.

[10] J. Breitenbach and J.L.J. Rosenfeld. Design of a Photogoniometer to Measure Angular Dependent Optical Properties. In Proceedings of International Conference on Renewable Energy Technologies in Cold Climates, pages 386-391, Ottawa, Canada, 1998. Solar Energy Society of Canada Inc.

[11] M. Andersen, L. Michel, C. Roecker, and J.-L. Scartezzini. Experimental assessment of bi-directional transmission distribution functions using digital imaging techniques. Energy and Buildings, 33(5):417-431, May 2001.

[12] M. Andersen, C. Roecker, and J.-L. Scartezzini. Design of a time-efficient video-goniophotometer combining bidirectional functions assessment in transmission and reflection. Solar Energy Materials and Solar Cells, 88(1):97-118, June 2005.

[13] J.H. Klems and J.L. Warner. Measurement of bidirectional optical properties of complex shading devices. In ASHRAE Transactions 101 (1), pages 791-801, Chicago, IL, 1995.

[14] P. Apian-Bennewitz and J. von der Hardt. Enhancing and calibrating a goniophotometer. Solar Energy Materials and Solar Cells, 54(1-4):309-322, August 1998. 
[15] M. Andersen. Innovative bidirectional video-goniophotometer for advanced fenestration systems. PhD thesis, EPFL, Lausanne, 2004.

[16] Commission Internationale de l'Eclairage. CIE Proceedings, 1931. Cambridge University Press, Cambridge, 1932.

[17] M. Andersen. Matrix-based analysis of digital images: application to goniophotometric measurements with variable referential. Optics and Lasers in Engineering, 43(3-5):419-435, March-May 2005. Special issue "Optics in Switzerland".

[18] M. Andersen and J.-L. Scartezzini. Inclusion of the specular component in the assessment of bidirectional distribution functions based on digital imaging. Solar Energy, 79(2):159-167, 2005. Special issue “CISBAT 2003”.

[19] Commission Internationale de l'Eclairage. Methods of characterizing illuminance meters and luminance meters - Performance, characteristics and specifications. CIE, 69, 1987.

[20] D. van Dijk. Daylighting Products with Redirecting Visual Properties (The project REVIS). Final Report JOE3-CT98-0096, TNO Building and Construction Research, Delft, February 2001.

[21] J.F. Murray-Coleman and A.M. Smith. The Automated Measurement of BRDFs and their Application to Luminaire Modeling. Journal of the Illuminating Engineering Society, 19(1):87-99, 1990.

[22] M. Andersen, J.-L. Scartezzini, C. Roecker, and L. Michel. Bi-directional Photogoniometer for the Assessment of the Luminous Properties of Fen- 
estration Systems. CTI Project 3661.2, LESO-PB/EPFL, Lausanne, May 2000.

[23] Commission Internationale de l'Eclairage. Practical methods for the measurement of reflectance and transmittance. CIE, 130, 1998.

[24] M. Andersen, M. Rubin, and J.-L. Scartezzini. Comparison between raytracing simulations and bi-directional transmission measurements on prismatic glazing. Solar Energy, 74(2):157-173, February 2003.

[25] M. Andersen, M. Rubin, R. Powles, and J.-L. Scartezzini. Bi-directional transmission properties of venetian blinds: Experimental assessment compared to ray-tracing calculations. Solar Energy, 78(2):187-198, 2005. 


\section{List of Tables}

1 Relative errors incurred at the different calibration stages and list of corresponding affected parameters in Equation (1). . . . . . . 30

2 Mean relative differences between TUB and EPFL measurements for the BTDF characterization of the opalescent acrylic for different incident altitudes $\theta_{1} \ldots \ldots \ldots \ldots \ldots \ldots$ 


\begin{tabular}{ccc}
\hline Error source & Relative error $(\%)$ & Affected parameter \\
\hline Spectral calibration & 0.6 & $L_{\text {screen }}$ \\
Photometric calibration & 1.0 & $L_{\text {screen }}$ \\
Illuminance measurement & 1.4 & $E_{1}$ \\
Beam collimation & 2.0 & $A$ \\
Image uniformity & 0.6 & $L_{\text {screen }}$ \\
Pixel resolution & 0.5 & $\left(\theta_{2}, \psi_{2}\right)$ \\
Eliminated pixels & 5.0 & $\mathrm{BT}(\mathrm{R}) \mathrm{DF}$ \\
Screen diffusion & 3.0 & $\rho_{\text {screen }}$ \\
Incident direction & 1.3 & $\left(\theta_{1}, \phi_{1}\right)$ \\
Screen position & 1.1 & $d^{2}$ \\
\hline
\end{tabular}




\begin{tabular}{|c|c|c|}
\hline$\theta_{1}\left(^{\circ}\right)$ & $\overline{\overline{\Delta B T D F}}_{\forall T D \theta_{2}}(\%)$ & $\frac{\overline{\Delta B T D F}}{B T D F} \theta_{2}<70^{\circ}(\%)$ \\
\hline 0 & 7.5 & 6.8 \\
\hline 12 & 8.9 & 8.2 \\
\hline 24 & 6.3 & 5.3 \\
\hline 36 & 6.2 & 5.0 \\
\hline 48 & 8.4 & 7.1 \\
\hline 60 & 6.7 & 5.1 \\
\hline 72 & 12.5 & 6.0 \\
\hline
\end{tabular}




\section{List of Figures}

1 Light detection principle in transmission (BTDF) and reflection (BRDF) modes for the LESO-PB / EPFL video-goniophotometer. 33

2 Photometric and geometric quantities for BT(R)DF definition. . 34

3 Conversion from $L_{\text {screen }}$ mappings into BT(R)DF data. . . . . . 35

4 Screen image: the edges of the elliptic covers generate a slight darkening of the pixels, which are therefore eliminated from the BT(R)DF assessment. . . . . . . . . . . . . . 36

5 BTDF data $\left(\mathrm{cd} \cdot \mathrm{m}^{-2} \cdot \mathrm{lx}^{-1}\right)$ monitored by TUB and EPFL for the sun directing glass, incidence $\left(\theta_{1}, \phi_{1}\right)=\left(60^{\circ}, 285^{\circ}\right)$; (a) Projection of BTDF data on virtual hemisphere: quasi-diffuse transmission sectors located in circles, (b) TUB photometric solid, (c) EPFL photometric solid. . . . . . . . . . . . . . . . 37

6 Directional-hemispherical light transmittance comparisons for diffusing and redirecting glazings: $\tau_{\mathrm{dh}}$ values based on BTDF integration compared to Ulbricht sphere measurements for (a) the white opalescent acrylic and (b) the Laser Cut Panel. . . . . . . 38

7 Directional-hemispherical light transmittance comparisons for a $3 \mathrm{M}$ prismatic film on float glass: $\tau_{\mathrm{dh}}$ values based on BTDF integration compared to Ulbricht sphere measurements for the $3 \mathrm{M}$ film on the (a) incidence side and (b) emerging side. . . . . . . . 39 


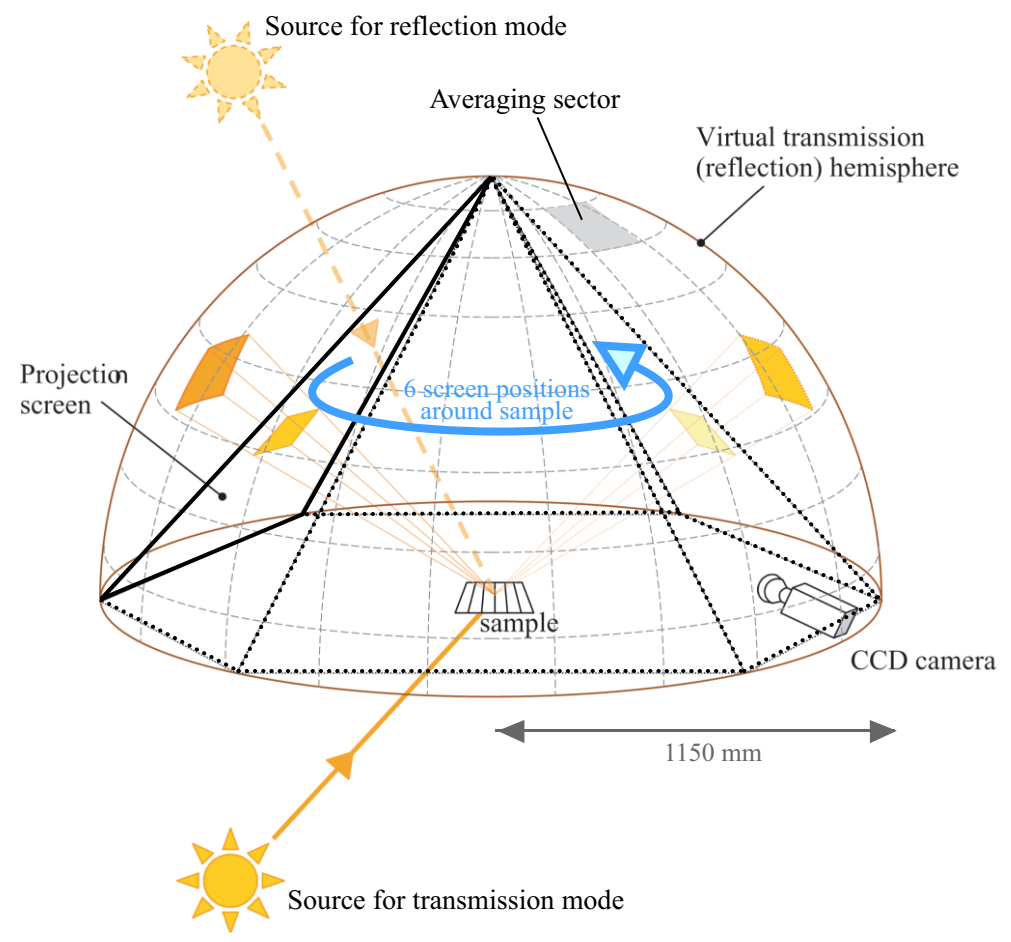





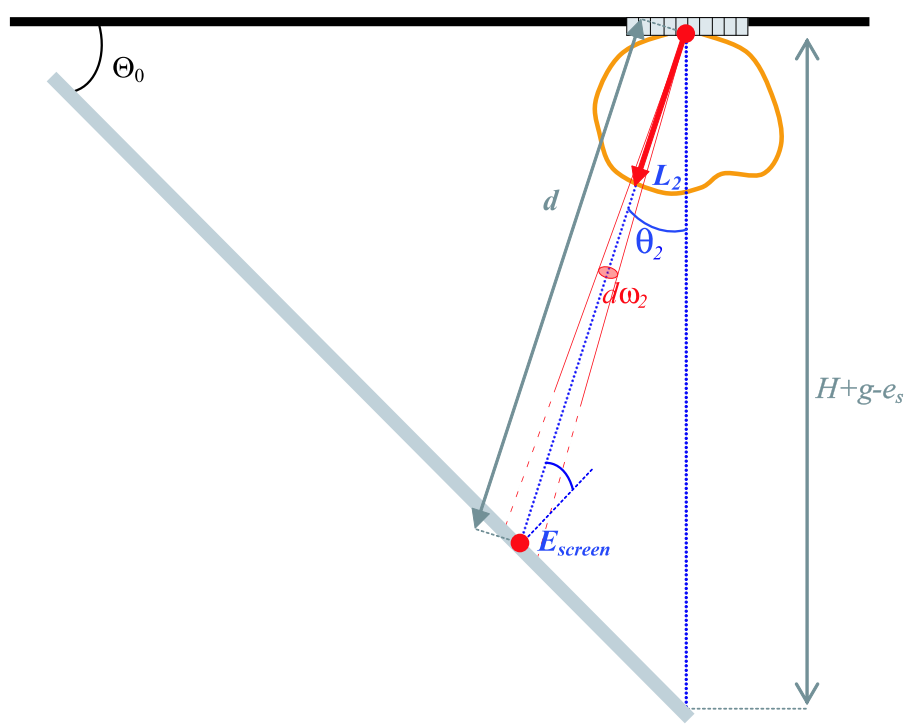




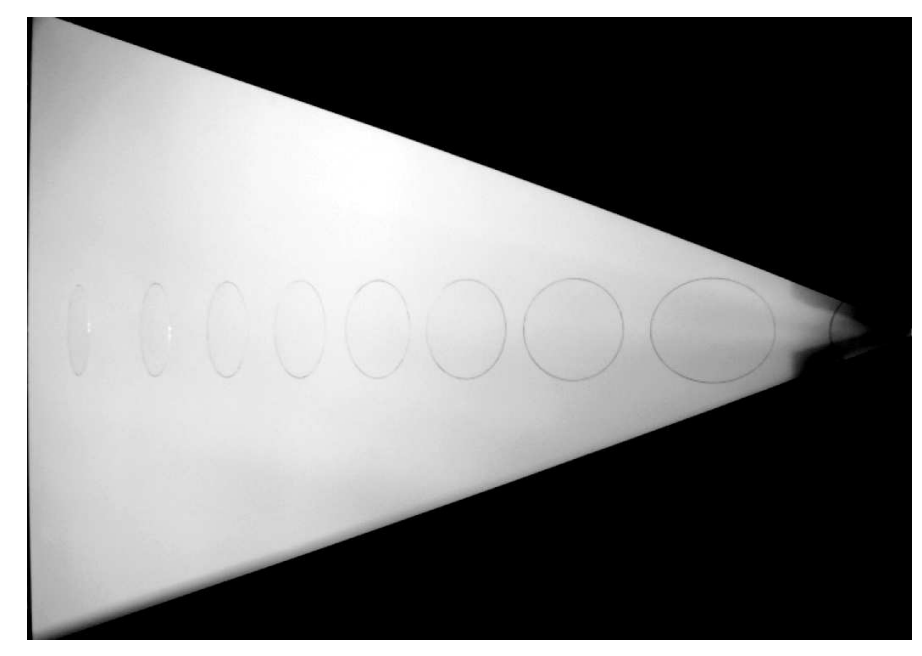



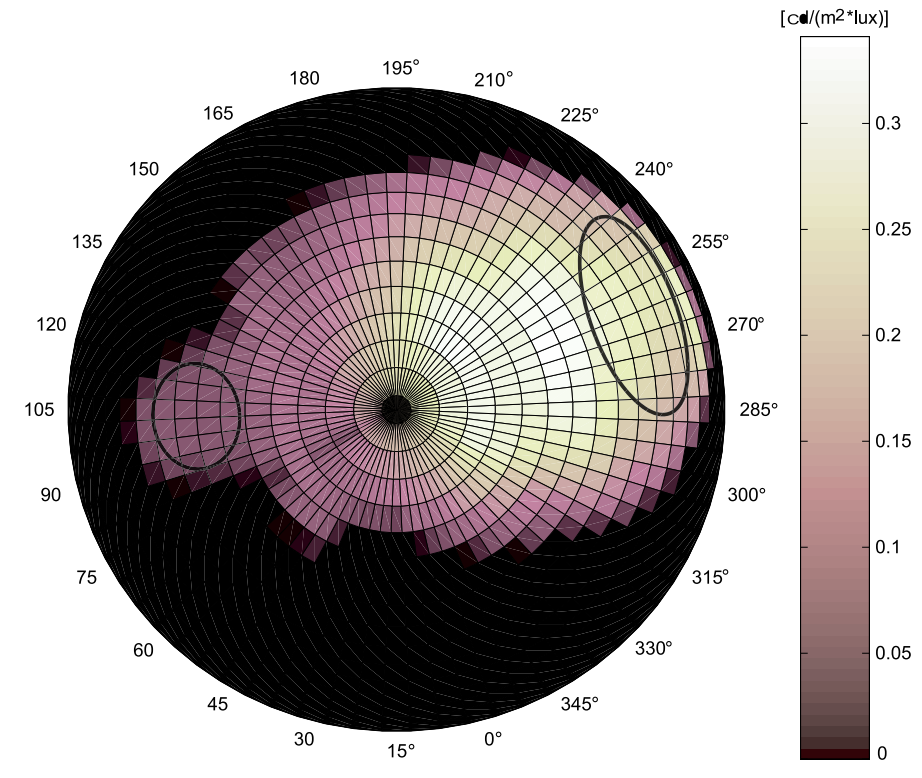

(a)

Bidirectional measurements of sun directing glass (Lumitop) light incidence $\varphi_{1}=285^{\circ}$ and $\theta_{1}=60^{\circ}$ (TUB)

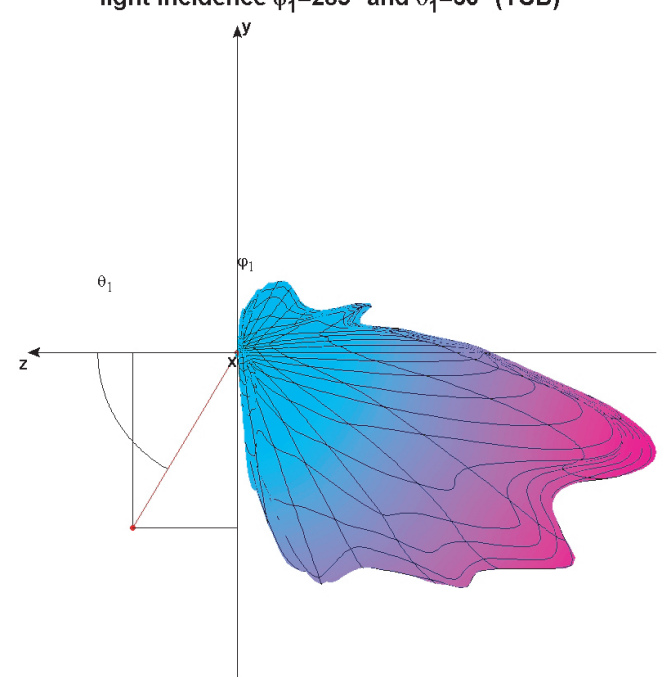

(b)

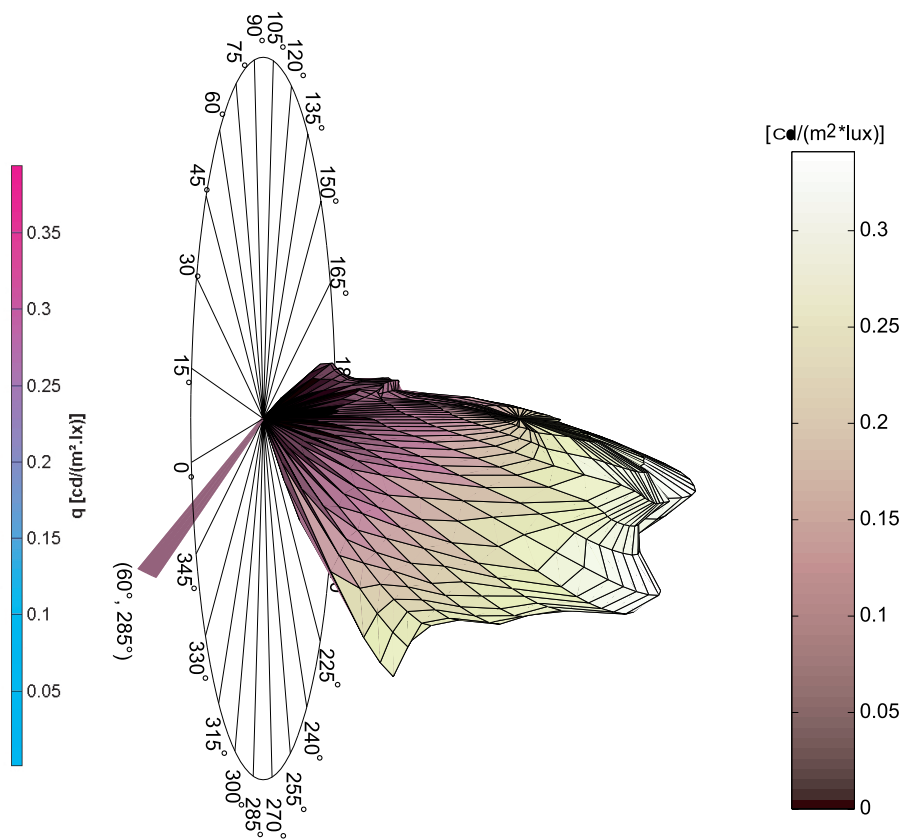

(c) 


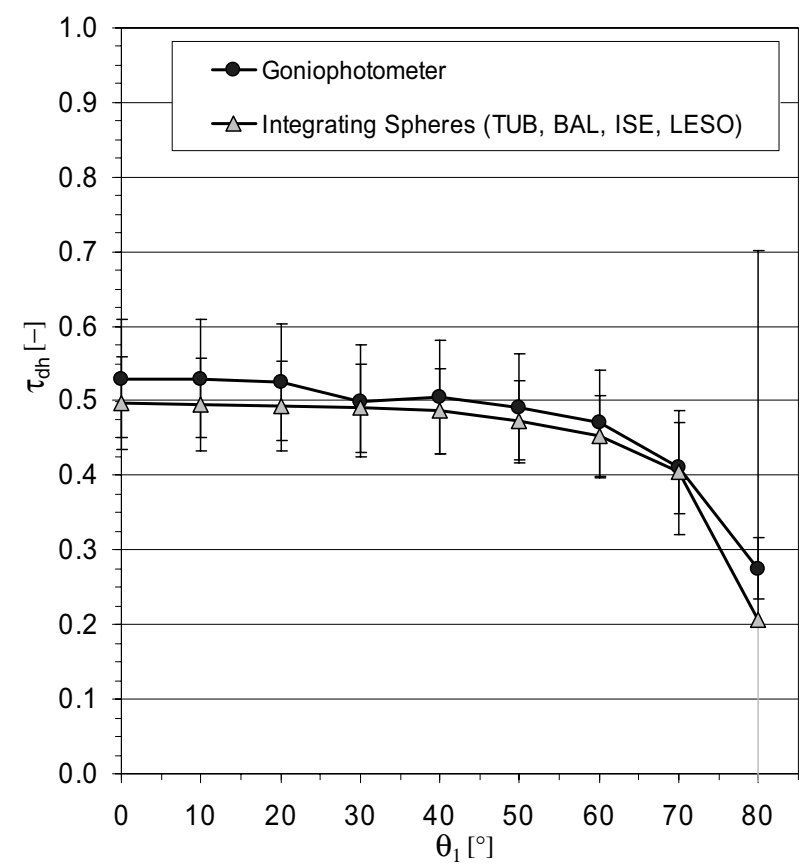

(a)

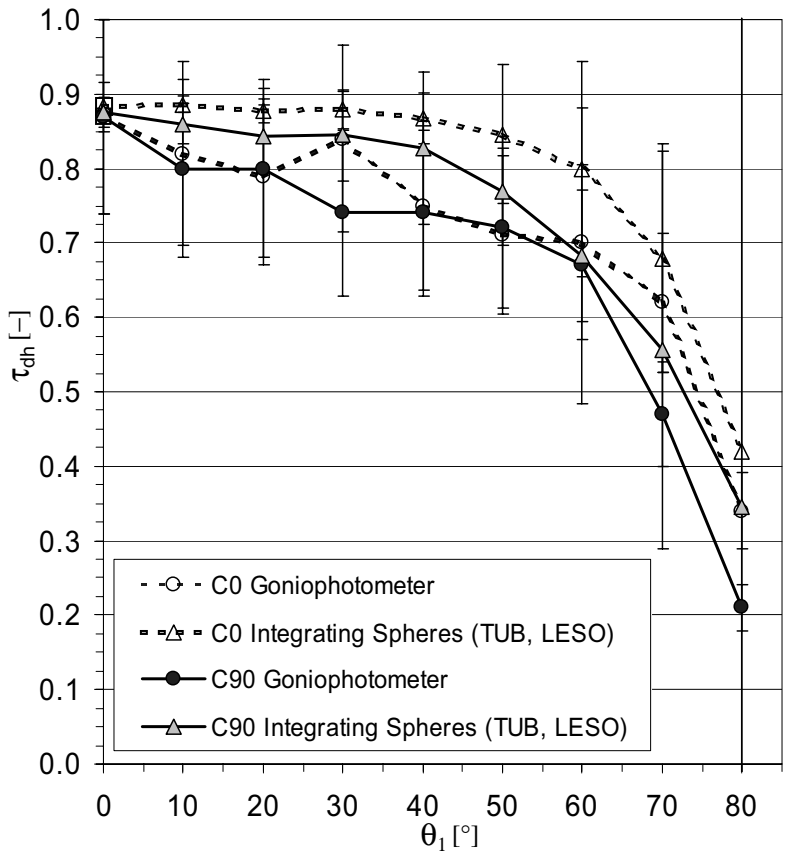

(b) 


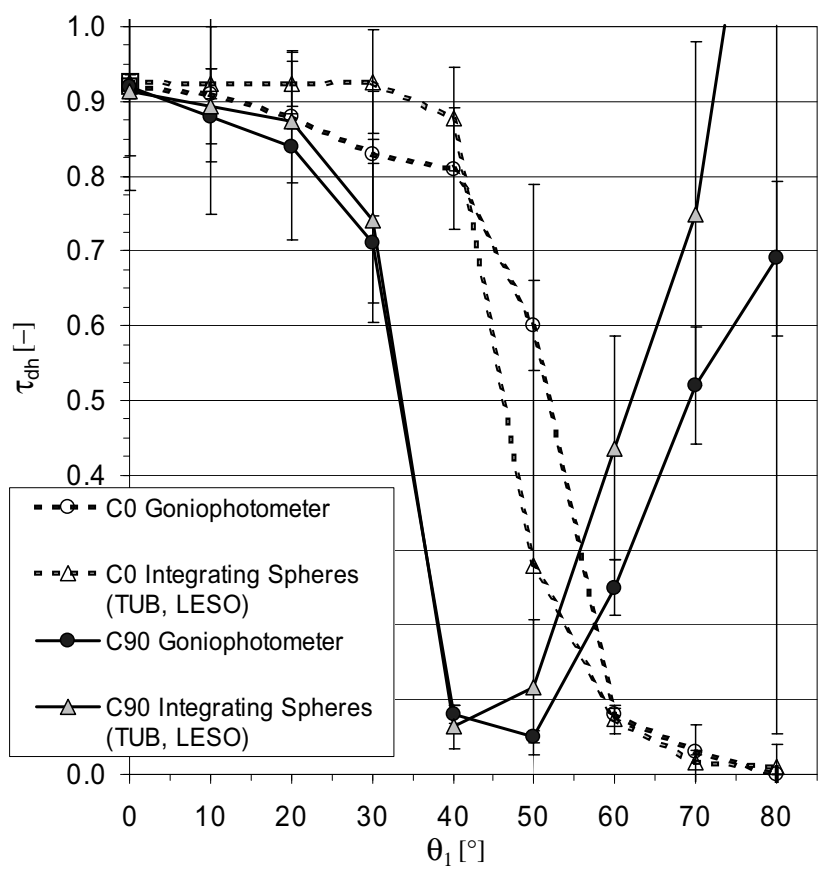

(a)

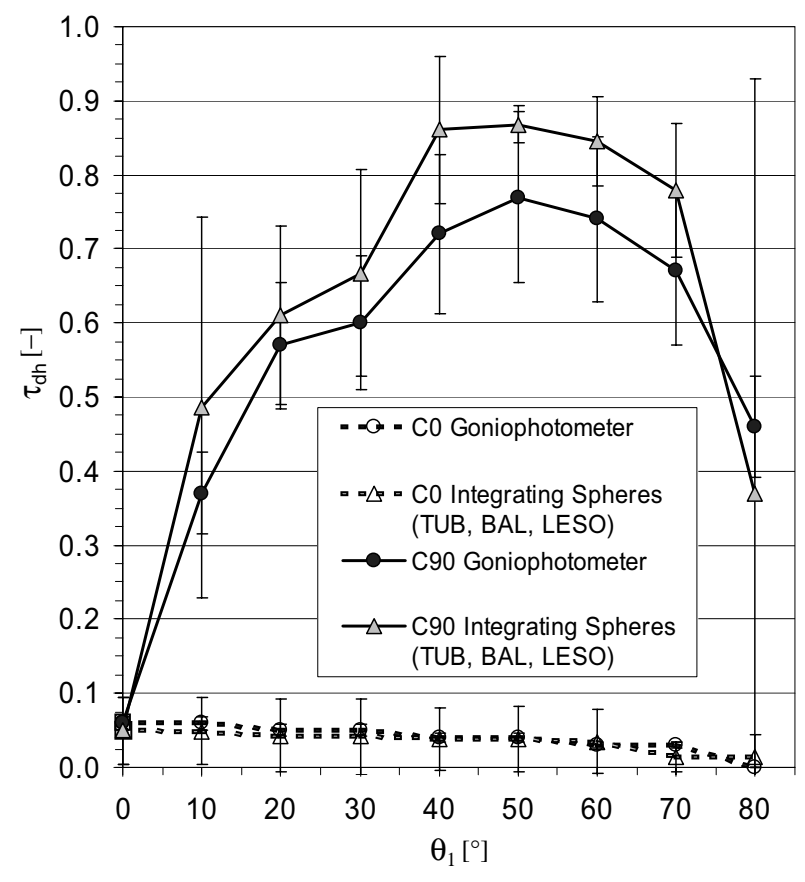

(b) 